\title{
Work-related variables associated with perceptions of recovery-oriented care among Quebec mental health professionals
}

Marie-Josée Fleury, Judith Sabetti, Guy Grenier, Jean-Marie Bamvita, Catherine Vallée and Zhirong Cao

\section{Background}

Provider working conditions are important in mental health service delivery.

\section{Aims \\ To identify variables associated with perceived recovery- oriented care among mental health professionals.}

\section{Method}

A total of 315 mental health professionals and 41 managers across four Quebec service networks completed questionnaires. Univariate and multilevel mixed-effects linear regressions for bivariate and multivariate analyses were performed using independent variables from the input-mediator-output-input model and recovery-oriented care.

\section{Results}

Recovery-oriented care related to: working in primary care or out-patient mental health services, team support, team interdependence, prevalence of individuals with suicide ideation, knowledge-sharing, team reflexivity, trust, vision (a subset of team climate), belief in multidisciplinary collaboration and frequency of interaction with other organisations.

\section{Conclusions}

Optimising team processes (for example knowledge-sharing) and emergent states (for example trust) may enhance recoveryoriented care. Adequate financial and other resources, stable team composition, training on recovery best practices and use of standardised assessment tools should be promoted, while strengthening primary care and interactions with other organisations.

\section{Declaration of interest}

None.

\section{Keywords}

Carers; community mental health teams; primary care; service users; recovery.

\section{Copyright and usage}

(c) The Royal College of Psychiatrists 2018. This is an Open Access article, distributed under the terms of the Creative Commons Attribution-NonCommercial-NoDerivatives licence (http://creativecommons.org/licenses/by-nc-nd/4.0/), which permits noncommercial re-use, distribution, and reproduction in any medium, provided the original work is unaltered and is properly cited. The written permission of Cambridge University Press must be obtained for commercial re-use or in order to create a derivative work.
International transformation to a recovery paradigm in mental health has involved major policy reform in high-income countries, including the UK and Canada. ${ }^{1}$ In Quebec, the 2005-2015 mental health reform ${ }^{2}$ defined recovery as personal empowerment, community integration and the inclusion of patients in decisionmaking. The reform aimed to create a well-integrated mental health system oriented towards recovery by strengthening primary care and out-patient services, and promoting various institutional links and partnerships among local service networks made up of diversified primary and specialised healthcare organisations. This major reform of the Quebec mental health system provided the backdrop for the study.

\section{Recovery-oriented care}

A number of structural elements and clinical best practices introduced in Quebec have been identified in other policy documents, and in research studies, as relevant to the ultimate goal of promoting personal recovery among mental health patients. Key elements include integrated service networks, ${ }^{3}$ shared-care initiatives involving the transfer of expertise from psychiatrists to primary care providers ${ }^{4}$ and community-based services offering better patientservice provider collaboration including individualised treatment planning. ${ }^{5}$ The Quebec reform also implemented known recoveryoriented best practices, such as strengths-based approaches, illness self-management and stepped care, ${ }^{6}$ while also promoting patient evaluation and feedback mechanisms, ${ }^{7}$ recovery education and training for mental health professionals, ${ }^{8}$ and more adequate funding. ${ }^{9}$

Support for multidisciplinary teams, and greater collaboration between mental health teams working in specialised mental health services and primary care settings, was a particular focus of the Quebec reform, and a policy interest shared by other countries, including the UK, for advancing recovery-oriented services. Wellresourced multidisciplinary teams that promote collaborative relationships over hierarchy and conflict have been shown to support the well-being and morale of mental health professionals and to meet the medical and psychosocial needs of individuals with mental disorders. ${ }^{10}$ Yet, tensions also arise in teams where a culture of recovery challenges the longstanding leadership of mental health professionals, notably psychiatrists and physicians accustomed to working from a medical-model perspective where they enjoy high status; ${ }^{11}$ tensions also occur when mental health professionals in multidisciplinary teams seek to retain their distinct professional roles in working with patients. ${ }^{12}$

\section{Existing research into recovery-oriented care}

A relatively small body of research has assessed mental health services in terms of recovery-oriented care. Studies have explored connections between socioprofessional characteristics of mental health clinicians, their working environments and recovery orientation; greater age, more professional experience and higher educational level related to recovery-oriented care. ${ }^{13-15}$ Professionals working in community-based services rated recovery orientation higher 
than their counterparts in hospital settings, even while controlling for age, civil status, education and job tenure. ${ }^{16}$ Regarding working conditions and job satisfaction, mental health professionals with lower job satisfaction scores expressed as emotional exhaustion and 'depersonalisation' (i.e. negative attitudes towards patients) produced lower scores on recovery-oriented care; whereas greater job and personal satisfaction produced higher scores. ${ }^{14,17}$ Organisational-level relationships were identified between strong leadership, larger budgets, and the perceived recovery orientation of services among mental health professionals, ${ }^{15}$ some of whom reported barriers to recovery implementation emanating from top-down organisational approaches, inadequate change management, staff resistance, deterioration in provider-manager relations and insufficient resources. ${ }^{18}$

Only three known studies have assessed teamwork, defined as 'the set of interrelated behaviors and actions that occur among team members while performing a task ${ }^{19}$ in relation to recoveryoriented care. Teamwork was identified as a facilitator for organisational transformation to recovery in an implementation study. ${ }^{20}$ A literature and policy review assessed challenges affecting multidisciplinary mental health teams responsible for developing individualised service plans for patients. ${ }^{21}$ In another study, mental health professionals rated early intervention teams as more recovery-oriented than adult mental health teams; and patient assessments revealed for the first time a strong association between recovery-oriented care provided by a team and their own personal recovery. $^{22}$

This incipient research points to a critical gap in knowledge: how the actual workings of mental health teams may relate to recovery-oriented care is virtually unknown. More specifically, team effectiveness depends on team processes (for example conditions such as knowledge-sharing and collaboration) and team-emergent states (for example motivation, cognition or emotions among team members, such as trust). ${ }^{19}$ These and other variables related to team effectiveness need to be investigated in association with recovery-oriented care. Moreover, we have very little understanding of how the larger organisational environment, or interactions between teams and mental health organisations, may inform the perceptions of mental health professionals regarding the recovery orientation of their work. The use of standardised assessment tools, which are known to improve team effectiveness, ${ }^{23}$ presents yet another line of inquiry for better understanding recoveryoriented care.

\section{Study aims}

The aim of this study was to identify and test a comprehensive range of variables related to professional characteristics, team characteristics, team processes, team-emergent states and organisational environment, most not previously examined, for associations with recovery-oriented care. The study also expands on previous research by developing a multidimensional conceptual framework rooted in both organisational science and clinical mental health practice. Based on theory and research related to recovery, and to team effectiveness, and given the strong focus on both elements in the Quebec mental health reform, we hypothesised that perceived recoveryoriented care among mental health professionals would be associated with: (a) out-patient (community or primary care) versus in-patient (hospital) settings; (b) team effectiveness, viewed in terms of multiple team processes (collaboration, knowledgesharing, etc) ${ }^{24}$ and team-emergent states (commitment to team, trust, etc); ${ }^{25}$ (c) greater service integration reflected in the frequency of team interactions with other organisations (for example community organisations, general practitioners (GPs)); and (d) the implementation of recovery best practices (for example illness self-management, stepped care) and standardised assessment tools (for example patient satisfaction, mental health/addiction evaluations).

\section{Method}

\section{Study design, sample and data collection}

This was a cross-sectional, multisite study. The setting included four local mental health service networks representing different geographic areas (urban and semi-urban), with or without a public psychiatric hospital and departments of psychiatry in general hospitals. Network populations ranged from 135000 to 300000 inhabitants. Each network also included multidisciplinary primary care teams staffed by GPs, allied health professionals and psychiatrists involved in shared-care and community organisations offering services such as crisis centres, day centres, rehabilitation programmes and selfhelp groups for mental health patients and families. Private medical clinics were also available.

Study participants were mental health professionals and managers employed in community-based or in-patient mental health services, or in primary care. To be eligible for the study, participants had to work in a multidisciplinary team consisting of three or more professionals from at least two disciplines in one of the four selected networks. All mental health professionals working in primary care or specialised mental health services who met these conditions, and their managers, were invited to participate.

Data collection occurred between October 2013 and June 2014. A total of 466 mental health professionals representing 49 mental health teams were invited to participate. Professionals completed a 222-item questionnaire that brought together 18 standardised scales covering multidimensional aspects of team effectiveness, with 9 separate questions on participant socioprofessional characteristics. Managers of the mental health professionals $(n=49)$ were also invited to participate in the study; they completed a separate questionnaire on: (a) patient profiles, (b) team composition, (c) use of recovery best practices, (d) use of evaluation procedures, (e) use of integration strategies, and (f) frequency and satisfaction with organisational and network interactions. Managers provided administrative information, but were not asked to evaluate recovery orientation. Questionnaires for mental health professionals and managers took 45 and $120 \mathrm{~min}$, respectively, to complete. A 12-member research advisory committee assisted with selection of the networks, recruitment and validation of the instruments; they also facilitated data collection. The research ethics board of a mental health university institute approved the research protocol.

\section{Conceptual framework, variables and instruments}

The conceptual framework for the study was adapted from the wellknown input-mediator-output-input (IMOI) team performance model $^{26}$ originating in occupational psychology (Fig. 1). Independent variables were organised within five conceptual blocks: professional characteristics, team characteristics, team processes, team-emergent states and organisational and territorial context. The IMOI model conceptualises individual variables (such as age, gender, profession) as nested within team-level variables (such as setting, composition), and, in turn, within the organisational context (such as structure, culture, interactions). ${ }^{25}$ The variables are viewed as so many inputs affecting team processes (actions directed at accomplishing tasks) and team-emergent states (cognitive, motivational or affective mediators), with 


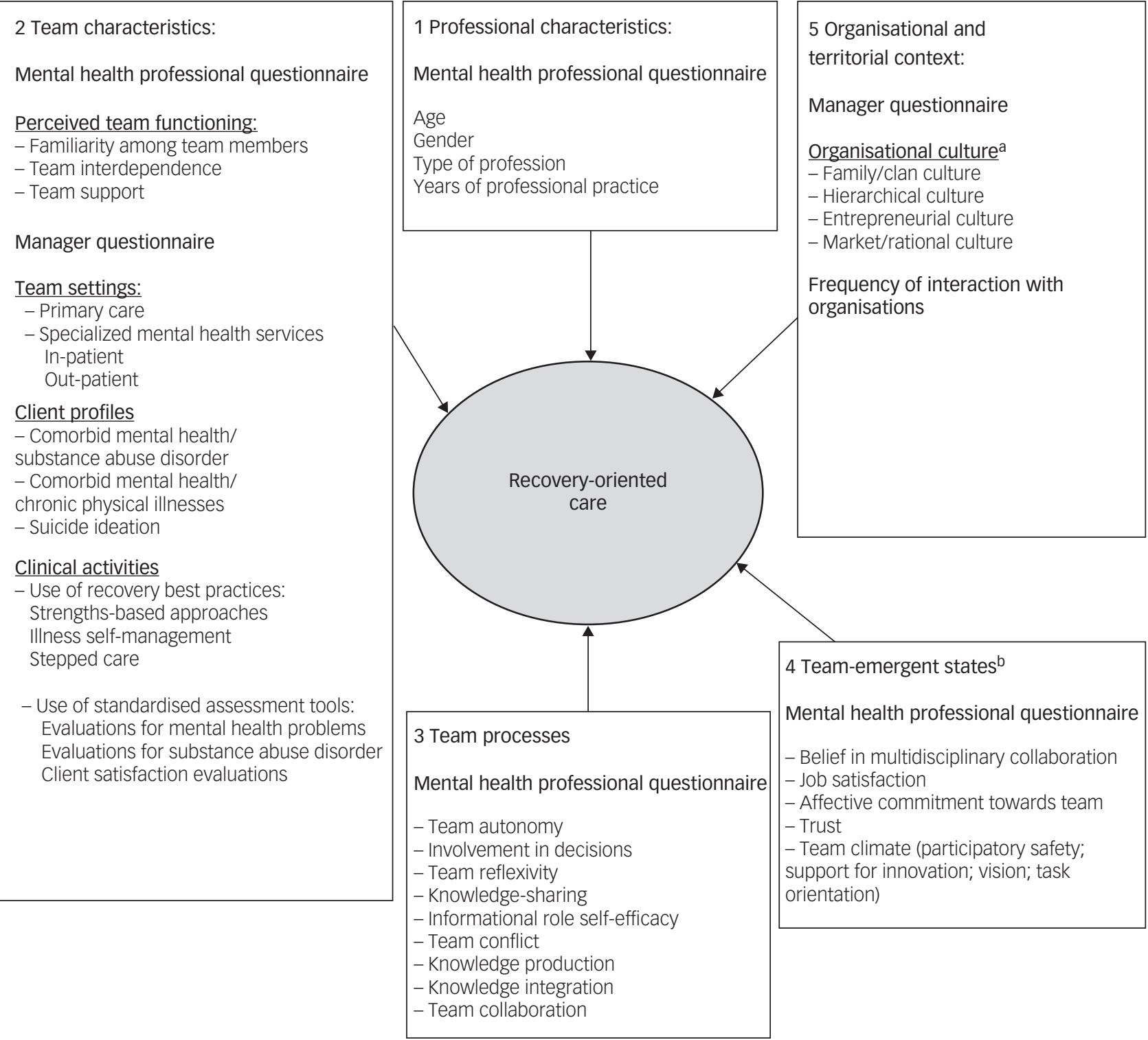

\section{Fig. 1 Conceptual framework}

${ }^{a}$ Scammon et al. ${ }^{27}$ describe the four cultures as follows: family/clan culture (internal focus, high employee involvement); authoritarian culture (stable, rule-based, efficient); entrepreneurial culture (external focus, innovative, risk tolerant); and market/rational culture (external focus, competitive, results oriented).

"Team "emergent states" are defined as elements that facilitate or impede the ability of team members to use their abilities in ways that generate the expected performance outcomes. $^{25}$

implications for the ability of team members to work together and produce desired results.

The dependent variable, recovery-oriented care, was measured with the Recovery Self-Assessment Scale (RSA) developed by O'Connell et al. ${ }^{28}$ The RSA is a 32 -item instrument based on six domains of recovery orientation: promoting life goals, consumer involvement, diversity of treatment options, consumer choice, individually tailored services and inviting environment, with scoring on a five-point Likert scale. Multiple versions of the RSA are available including the version for service providers and administrators used with mental health professionals in this study. The RSA has demonstrated good internal consistency, test-retest reliability, and convergent and discriminant validity; ${ }^{16,28,29}$ it is the most widely used instrument for assessing the recovery orientation of mental health services. ${ }^{30}$ Mental health professionals were invited to respond to questions on the RSA in terms of the care provided by their respective teams. Details on the RSA and the other 17 standardised instruments used to measure independent variables in the study, including their reliability measures, are presented in Table 1.

\section{Data analysis}

Since missing values were fewer than $5 \%$, they were replaced by the variable means. Normality assumptions were assessed for the dependent variable, recovery-oriented care, which was found to be normally distributed. Bivariate and multivariate analyses were conducted using multilevel mixed-effects linear regression to account for both fixed effects and random effects for clustering at the team level. The Akaike Information Criterion (AIC) was used to compare multiple linear regression models. Forward selection was performed for the mode selection based on the variables 
Table 1 Description of standardised instruments included in the study

Cronbach's alpha

Measures and descriptions

coefficients

Dependent variable

(1) Recovery-oriented care, ${ }^{28} 32$ items, 5 subscales

$0.76-0.90$ Independent variables

(2) Familiarity among team members; ${ }^{24} 5$ items

$0.78-0.88$

(3) Team interdependence, ${ }^{31} 20$ items

(4) Team support; ${ }^{32} 4$ items

(5) Team autonomy; ${ }^{33} 3$ items

(6) Involvement in decisions, ${ }^{33} 3$ items

(7) Team reflexivity; ${ }^{34} 3$ items

(8) Knowledge-sharing:; 5 items

(9) Informational role self-efficacy; ${ }^{36} 5$ items

(10) Team conflict; ${ }^{37} 9$ items

(11) Knowledge production; ${ }^{35} 5$ items

(12) Knowledge integration; 389 items

(13) Team collaboration; 39 items

(14) Belief in multidisciplinary collaboration; ${ }^{40} 5$ items

(15) Job satisfaction; ${ }^{41} 20$ items, 5 subdimensions

(16) Affective commitment towards team; ${ }^{42} 5$ items

(17) Trust; 434 items

(18) Team climate; ${ }^{44} 19$ items; 4 subdimensions
$0.77-0.88$

$0.84-0.85$

0.76

0.88

0.79

0.93

0.93

$0.93-0.94$

0.95

0.95

$0.77-0.91$

0.92

$0.60-0.82$

$0.86-0.92$

0.90

$0.60-0.84$

identified as significant (with alpha set at 0.10 ) in the bivariate analyses. The model with the smallest AIC was chosen for the multivariable analysis. Stata version 15 was used to conduct the analyses.

\section{Ethics approval and consent to participate}

All procedures performed in this study involving human participants were in accordance with the ethical standards of the institutional committee and with the 1964 Helsinki declaration and its later amendments or comparable ethical standards. Informed consent was obtained from all participants, each of whom signed a consent form. The multisite study protocol and consent form were approved by the Ethics Board of the Douglas Mental Health University Institute: MP-IUSMD-11-037.

\section{Results}

Of the 466 professionals invited to the study, 315 (67.60\%) from 49 teams participated. There were no significant differences between participants and non-participants on distributions for team setting $\left(\chi^{2}(1, n=466)=0.79 ; P=0.68\right)$, or gender $\left(\chi^{2}(1, n=466)=\right.$ $0.03 ; P=0.87)$. Mean age was 43 years and the mean tenure on teams was 3 years. Women outnumbered men by more than two to one $(70 \% v .30 \%)$. In total, $56 \%$ worked in out-patient mental health services; $32 \%$ in primary care and $12 \%$ in in-patient mental health services.

Regarding managers, 41 of 49 (84\%) participated in the study. Their mean age was 44 years, and $71 \%(n=29)$ were women; $63 \%$ $(n=26)$ worked in specialised mental health services and $37 \%$ $(n=15)$ in primary care, with an average seniority of 4 years. Comparison analyses revealed no differences between respondent and non-respondent managers on gender (Pearson $\chi^{2}=0.966$; d.f. $=1$; Fisher's exact test two-sided $P=0.663$ ) or team setting (Pearson $\chi^{2}=1.861$; d.f. $=2$; Fisher's exact test two-sided $P=0.245$ ).

Analyses were conducted for 312 participants from 47 teams, after 3 participants from two teams were eliminated because of small team size $(n<3$, and a mean of 6.64 observations per team (range 3-16)). There was an effect of clustering at the team level (intraclass correlation coefficient $15.00 \%$, 95\% CI 6.56-30.62). The dependent variable, recovery-oriented care, had a mean score of 3.64 (range: $1.54-4.89$, s.d. $=0.49$ ) and was normally distributed (Skewness: -0.50 ; Kurtosis: 4.20).

Variables significantly associated with recovery-oriented care in the bivariate analysis (Table 2), were used to build the multiple linear regression model (Table 3). Eleven variables were identified in the multiple regression model as significantly and positively related to recovery-oriented care. They included five variables from the team characteristics block: the two team settings variables (primary care versus in-patient mental health services; and outpatient mental health services versus in-patient mental health services), which confirms our first hypothesis, and three other team characteristics variables: team interdependence, team support and individuals with suicide ideation. Variables from the team processes block included knowledge-sharing, and team reflexivity, which was marginally associated with the dependent variable. Within teamemergent states, belief in multidisciplinary collaboration, trust and vision, a subset of team climate, emerged as associated with recovery-oriented care, confirming our second hypothesis. Finally, under organisational and territorial context, frequency of interaction with other organisations was associated with recovery-oriented care, which seems to confirm our third hypothesis.

\section{Discussion}

\section{Main findings}

This study identified 11 variables in relation to recovery-oriented care in mental health services across four of the five conceptual blocks in our adapted IMOI framework, as follows: team characteristics (5 variables), team processes (2 variables), team emergent states (3 variables), and organisational and territorial context (1 variable). Only the provider characteristics block failed to produce significant findings, likely as a result of the effects of introducing a large number of team processes and team-emergent states variables into the model. We may presume that team processes and team-emergent states were linked to recovery-oriented care by a shorter causal chain than provider characteristics, and that they had a more direct influence. ${ }^{45}$ Results for the global score on recovery-oriented care (mean: 3.6, s.d. $=0.49$ ) were comparable with those obtained by the authors of the RSA, ${ }^{28}$ and nearly identical to results reported in Leamy et $a l^{22}$ which evaluated recovery orientation among different types of teams.

\section{Community care}

Our first hypothesis, that mental health professionals working in primary care or in out-patient mental health services would rate recovery-oriented care in their services higher than professionals working in in-patient (hospital) settings, was confirmed. This result reaffirms the basic recovery principle that mental health services should be anchored in the community, and lends particular support to studies suggesting that primary care or out-patient mental health services are more normalising, or less stigmatising, for patients, ${ }^{46}$ as well as for professionals. By contrast, professionals whose experience with mental health patients occurs largely in in-patient settings may overlook, or even disavow, the possibility of recovery. ${ }^{47}$ Another explanation for this finding may be that in-patient mental health services lack the biopsychosocial approach characteristic of community-level services such as primary care, especially in treating mental health patients whose risk factors for comorbid substance use disorders, as well as physical morbidity and mortality, are very high.

\section{Team support}

Financial and material support, key components of team support, is another positive factor in recovery-oriented services, and was found to be associated with larger per capita budgets. ${ }^{15}$ This 


\section{Professional characteristics}

Gender, $n$ (\%)

Women

$216(69.23)$

Type of profession, $n$ (\%)

Medical professions

Psychosocial professions

General professions

Age, years: mean (s.d.)

Years of professional practice, mean (s.d.)

Team characteristics

Team settings, $n$ (\%)

Primary care

Out-patient mental health services

In-patient mental health services

Perceived team functioning, mean (s.d.)

Familiarity among team members

Team interdependence

Team support

Patient profiles, mean (s.d.)

Comorbid mental health/substance use disorder

Comorbid mental health/chronic physical illness

suicide ideation

Clinical activities, mean (s.d.)

Strengths-based approaches

Evaluation for mental health problems

Evaluation for substance use disorder problems

Patient satisfaction evaluations

Team processes, mean (s.d.)

Team autonomy

Involvement in decisions

Team reflexivity

Knowledge-sharing

Informational role self-efficacy

Team conflict

Knowledge production

Knowledge integration

Team collaboration

Team-emergent states, mean (s.d.)

Belief in multidisciplinary collaboration

Job satisfaction

Affective commitment towards team

Trust

Team climate: participatory safety

Team climate: support for innovation

Team climate: vision

Team climate: task orientation

Organisational and territorial context, mean (s.d.)

Frequency of interaction with organisations

Organisational culture

Family/clan culture

Entrepreneurial culture

Market/rational culture

Hierarchical culture

Results in bold are significant.
$96(30.77)$

109 (34.94)

$170(54.49)$

$33(10.58)$

$43.33(10.51)$

9.06 (10.84)

101 (32.37)

$174(55.77)$

37 (11.86)

$5.37(0.91)$

$4.56(1.04)$

4.83 (1.17)

$34.24(23.00)$

34.24 (21.69)

28.08 (19.87)

$3.29(1.06)$

$4.11(1.46)$

3.60 (1.17)

$3.10(1.28)$

$4.92(1.26)$

5.01 (1.36)

$4.58(1.25)$

$5.73(0.90)$

81.01 (14.43)

$3.00(0.97)$

3.99 (1.23)

$4.25(1.13)$

$4.82(0.96)$

$6.23(0.73)$

4.95 (0.72)

4.85 (1.23)

$5.19(1.16)$

$5.16(0.95)$

$5.15(1.05)$

$5.05(0.96)$

5.09 (1.00)

$-0.026$

0.668

$-$

0.013

$-0.048$

$-0.001$

$-0.002$

0.834

0.614

0.664

0.415

0.222

0.314

0.064

0.006

$-$

0.176

0.172

0.164

$<0.001$

0.001

0.004

0.048

0.048

0.035

0.056

0.078

0.154

0.155

0.231

0.010

$-0.106$

0.168

0.182

0.219

0.195

0.249

0.131

0.183

0.229

0.221

0.241

0.181

0.004

$-0.001$

$<0.001$

0.002

$<0.001$

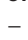

$<0.001$

$<0.001$

$<0.001$

0.882

0.487

0.036

0.174

$\mathbf{0 . 0 5 3}$

0.272

0.048

$<0.001$

$<0.001$

$<0.001$

$<0.001$

$<0.001$

$<0.001$

$<0.001$

$<0.001$

$<0.001$

$<0.001$

$<0.001$

$<0.001$

$<0.001$

$<0.001$

$<0.001$

$<0.001$

$<0.001$

$<0.001$

0.236

0.879

$\mathbf{0 . 0 8 3}$

0.971 stands to reason, as recovery practices are highly individualised, labour intensive and complex in terms of service delivery. The preponderance of financial and material components in the team support variable (team characteristics), confirms this and indirectly supports the contention that insufficient financial resources impede recovery implementation in mental health systems. ${ }^{18}$ However, 'human' or clinical support, the fourth element in the team support measure, was also crucial. Research confirms that clinical supervision plays a major role in controlling dimensions related to the work environment, such as interpersonal relationships within teams, the integration of good practices and other innovations that help alleviate the stress of frequent patient interactions, and protect mental health professionals against burnout. $^{48}$

\section{Team interdependence}

To our knowledge, studies to date have not assessed the relationship between team interdependence and recovery-oriented care; yet the positive association identified in this study seems reasonable. A high level of interdependence among mental health professionals working as team members positively influences team 


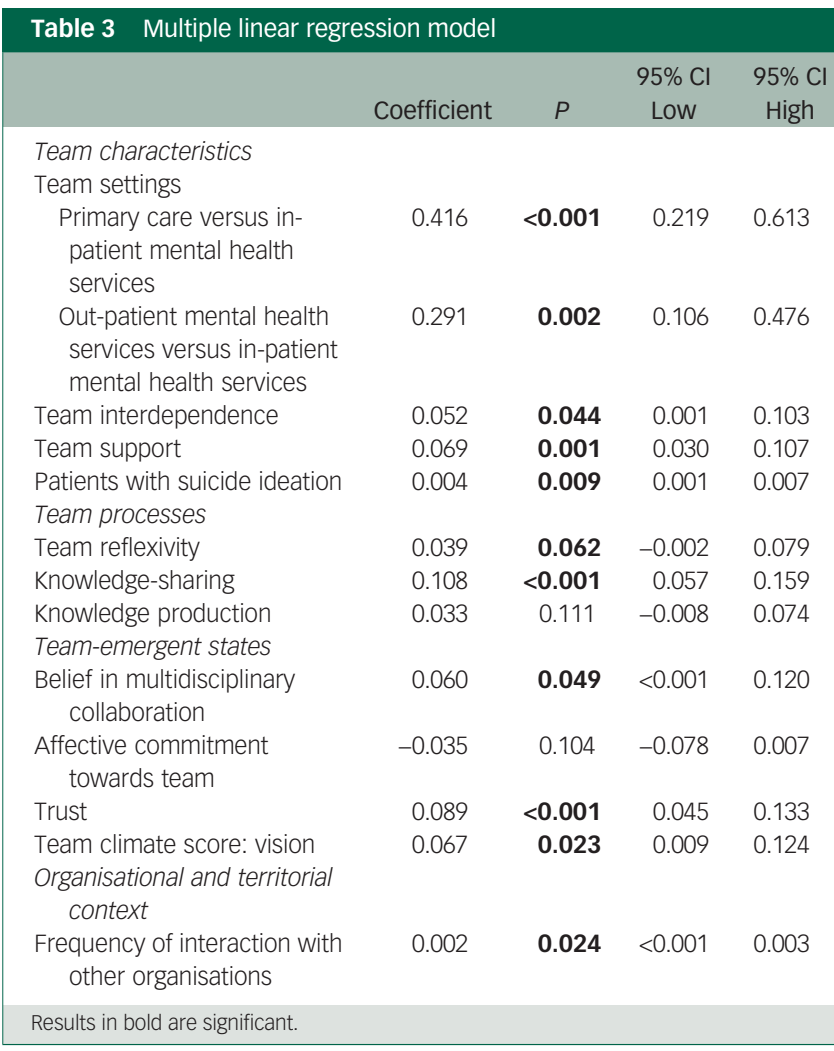

effectiveness, ${ }^{25}$ which should have a bearing on their capacity to deliver recovery-oriented care. Interdependence is particularly important in mental health teams, which require mental health professionals to collaborate in a multitude of tasks. ${ }^{49}$ Mental health professionals also need to transcend the boundaries of their disciplines in order to provide person-centred biopsychosocial services and integrated $_{\text {care. }}{ }^{11}$

\section{Knowledge sharing and reflexivity in teams}

Our second hypothesis on a possible association between favourable aspects of team processes and emergent states and recoveryoriented care was also confirmed. Concerning knowledge-sharing and reflexivity in teams (team processes), and recovery-oriented care, our results partially support those of a recent study, ${ }^{50}$ which found that knowledge integration was significantly related to patient-centred services in teams; knowledge-sharing was, in turn, a necessary first step before undertaking knowledge integration. Reflexivity, or 'the extent to which team members overtly reflect upon the group's objectives, strategies, and processes and adapt them to current or anticipated endogenous or environmental circumstances $^{34}$ was associated with team learning. Moreover, studies indicate that reflexivity and knowledge-sharing are both associated with team interdependence. ${ }^{34}$

\section{Trust and shared vision and beliefs}

Similar to our finding on trust (team emergent states) and recoveryoriented care, other research has demonstrated that mutual trust is a significant protective factor against emotional exhaustion, depersonalisation and burnout among mental health professionals, factors inversely related to recovery orientation. ${ }^{17}$ Furthermore, vision refers to grand objectives that heighten motivation among team members. ${ }^{44}$ The higher the vision, the more professionals generally strive to achieve their objectives. Not surprisingly, a clear and shared vision focused on recovery orientation favours the implementation of relevant values and practices. ${ }^{51}$ Finally, a strong facilitating factor in multidisciplinary collaboration involves a belief in its accrued benefits for various professionals and their patients. $^{12}$

\section{Interaction between mental health teams and other organisations}

Our third hypothesis on the value of frequent interaction between mental health teams and other organisations for recovery-oriented care was confirmed. Such interactions allow mental health teams to influence the values and skills of their organisational partners, facilitating the diffusion of a recovery approach to local service networks. ${ }^{13,20}$ The inability of single teams to provide the full range of services essential to patient recovery underscores the importance of interorganisational interactions from a shared recovery perspective. ${ }^{20}$ By the same token, the lack of collaboration among health providers emerged as an important barrier to the integration of recovery-oriented care. ${ }^{18}$

In Quebec, the nature of collaboration between public and community organisations reflects a systems-focused management style that seems to auger well for implementing a recovery orientation in services. The 2005 mental health reform mandated greater integration within local service networks, and a more comprehensive response to patient needs, particularly increased collaboration between public and community organisations. Among public organisations, for example, addiction rehabilitation centres are working increasingly with mental health teams in response to the needs of individuals with co-occurring disorders. Other integration strategies include cross training, service agreements and deployment of addiction liaison nurses in hospital emergency rooms to ensure more timely and appropriate patient referral. ${ }^{4}$ Community organisations play another crucial role, providing a normalising context for the delivery of primary healthcare services to deinstitutionalised individuals, while addressing their basic subsistence needs for housing, employment and food. Community organisations promote personal empowerment, mutual help and the creation of social networks.

\section{Use of standardised assessment tools}

Our fourth hypothesis proposed a relationship between the implementation of recovery best practices and standardised assessment tools and recovery-oriented care. Surprisingly, no results emerged in the model for the various recovery-oriented best practices and standardised assessment tools tested. We might presume that further work was still needed in the networks to more fully integrate these measures, at the time the research was conducted. Another study has critiqued the Quebec reform for focusing on structural changes, without providing sufficient guidelines on the use of clinical approaches or standardised assessment tools, ${ }^{52}$ which may explain why they were perhaps underused or took longer than expected to implement in services.

\section{Suicidal ideation and $24 \mathrm{~h}$ crisis services}

Finally, a significant finding emerged outside of the four study hypotheses, namely the positive relationship between higher proportions of individuals with suicide ideation on case-loads (team characteristics) and recovery-oriented care. This finding is not surprising, as mental health professionals working within a recovery philosophy expect to provide intensive, personalised service adapted to the needs, and uneven recovery process, of individuals with suicide ideation. As well, mental health professionals in Quebec working with patients who were suicidal were able to avail themselves of additional support from the $24 \mathrm{~h}$ crisis services offered by community organisations. Relationships between mental 
health professionals working in Quebec mental health teams and the staff from community crisis services were found to be particularly strong, ${ }^{53}$ suggesting that crisis services have benefits for service providers as well as clients. The availability of $24 \mathrm{~h}$ crisis services is viewed as an important element in recovery-oriented care. ${ }^{54}$

\section{Limitations}

This study had limitations that should be acknowledged. First, as this is a cross-sectional study, causal inferences about the findings cannot be made. Second, the study measured provider perceptions of recovery-oriented care, without making links between recoveryoriented care and patient outcomes in terms of their personal recovery. Third, the results of this study based on data from four Quebec regions need to be replicated in other settings, and the perspectives of patients sought.

\section{Implications}

This study was the first to test a comprehensive set of variables related to team characteristics, processes, team-emergent states and interorganisational relationships for associations with recovery-oriented care from the perspectives of mental health professionals. The findings coincide with an incipient, but promising, body of research on provider issues in relation to recovery-oriented care. The study is original in focusing attention on team effectiveness as a topic of concern for the recovery field, where patient issues and outcomes have tended to take precedence over workrelated conditions affecting those responsible for service delivery. Future research should continue to focus on team and provider effectiveness, within the wider operational environment in mental health systems, as an important pathway for realising conditions more favourable to mental health recovery.

Findings confirm the greater recovery orientation of primary care and out-patient mental health services over in-patient mental health services, and the importance of building relationships among mental health professionals working in various public and community organisations within local service networks. The study suggests that primary care services and mental health out-patient services should be strengthened in response to the needs of mental health professionals aiming to provide effective biopsychosocial services for mental health patients living in the community, particularly indviduals with suicide ideation. Greater integration between primary care and specialised mental health services may also support enhanced recovery orientation in teams, as occurred in the collaboration between mental health teams and community organisations in this study. Team managers should foster the diffusion of a clear and shared vision of recovery, as well as the flexibility and openness needed to support mental health recovery among patients. Other findings established the significance of knowledge-sharing, reflexivity, interdependence, support and trust as attributes of teams that support greater recovery-oriented care.

Our study suggests that mental health decision-makers should focus on optimising team processes and team-emergent states for enhancing recovery-oriented care in mental health teams. Financial, material and human resources must also be adequate to support team working conditions favourable to recovery-oriented care, as well as good clinical supervision. Stable team composition may facilitate the creation of trust among team members; and a clear vision favours implementation of innovative recovery-based practices. Finally, teams would be further strengthened if mental health professionals were better trained and supported to adopt recovery best practices and to make use of standardised assessment tools that reflect a recovery approach to service delivery.
Marie-Josée Fleury, PhD, Professor, Department of Psychiatry, McGill University and Researcher, Douglas Mental Health University Institute Research Centre, Canada; Judith Sabetti, PhD, Adjunct Professor, McGill University School of Social Work and Research Agent, Douglas Mental Health University Institute Research Centre, Canada; Guy Grenier, PhD, Research Associate, Douglas Mental Health University Institute Research Centre, Canada; Jean-Marie Bamvita, MD, PhD, Research Agent, Douglas Mental Health University Institute Research Centre Canada; Catherine Vallée PhD

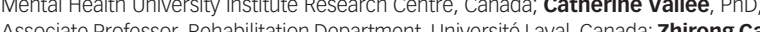
Associate Professor, Rehabilitation Department, Universite Laval, Canada, Zhirong Ca, MA, Research Agent, Douglas Mental Health University Institute Research Centre, Canada

Correspondence: Marie-Josée Fleury, PhD, Douglas Mental Health University Institute Research Centre, 6875 LaSalle Blvd., Montreal H4H 1R3, QC, Canada. Email: flemar@ douglas.mcgill.ca

First received 5 Jan 2018, final revision 3 Oct 2018, accepted 5 Oct 2018

\section{Funding}

This study was funded by the Fonds de la recherche en santé du Québec (FRSQ), grant number 22367 and the Prends soin de toi Program.

\section{Acknowledgements}

We would like to thank the funding agencies cited above for their support, as well as the individuals who participated in this research, our research advisory committee, network respondents; and many thanks to Denise Aubé, and François Chiocchio for their valuable assistance.

\section{References}

1 Adams N, Daniels A, Compagni A. International pathways to mental health transformation. Int J Ment Health 2009; 38: 30-45.

2 Ministère de la Santé et des Services sociaux (MSSS). Plan D'action en Santé Mentale 2005-2010 : La Force des Liens [Mental Health Action Plan, 20052010: The Strength of Relationships]. MSSS, 2005.

3 Fleury MJ. Integrated service networks: the Quebec case. Health Serv Manage Res 2006; 19: 153-65

4. Fleury MJ, Grenier G, Vallée C, Aubé D, Farand L, Bamvita JM, et al. Implementation of the Quebec Mental Health Reform (2005-2015). BMC Health Serv Res 2016; 16: 228-40.

5 Stanhope V, Ingoglia C, Schmelter B, Marcus SC. Impact of person-centered planning and collaborative documentation on treatment adherence. Psychiatr Serv 2013; 64: 76-9.

6 Menear M, Briand C. Implementing a continuum of evidence-based psychosocial interventions for people with severe mental illness: part 1 - Review of major initiatives and implementation strategies. Can J Psychiatry 2014; 59: 178-86.

7 Marshall SL, Oades LG, Crowe TP. Australian mental health consumers' contributions to the evaluation and improvement of recovery oriented service provision. Isr J Psychiatry Relat Sci 2010; 47: 198-205.

8 Salgado JD, Deane FP, Crowe TP, Oades LG. Hope and improvements in mental health service providers' recovery attitudes following training. $J$ Ment Health 2010; 19: 243-8.

9 Salkever D, Gibbons B, Ran X. Do comprehensive, coordinated, recoveryoriented services alter the pattern of use of treatment services? Mental health treatment study impacts on SSDI beneficiaries' use of inpatient, emergency, and crisis services. J Behav Health Serv Res 2014; 41: 434-46.

10 Malinovsky I, Lehrer P, Silverstein SM, Shankman SA, O'Brien W, Samuelson T, et al. An empirical evaluation of recovery transformation at a large community psychiatric rehabilitation organization. Psychol Serv 2013; 10: 428-41.

11 Maddock A. Consensus or contention: an exploration of multidisciplinary team functioning in an Irish mental health context. Eur J Soc Work 2015; 18: 246-61.

12 Supper I, Catala O, Lustman M, Chemla C, Bourgueil Y, Letrilliart L. Interprofessional collaboration in primary health care: a review of facilitators and barriers perceived by involved actors. J Public Health 2015; 37: 716-27.

13 McLoughlin KA, Fitzpatrick JJ. Self-reports of recovery-oriented practices of mental health nurses in state mental health institutes: development of a measure. Issues Ment Health Nurs 2008; 29: 1051-65.

14. Osborn LA, Stein $\mathrm{CH}$. Mental health care providers' views of their work with consumers and their reports of recovery-orientation, job satisfaction, and personal growth. Community Ment Health J 2015; 52: 757-66.

15 Brown TT, Mahoney CB, Adams N, Felton M, Pareja C. What predicts recovery orientation in county departments of mental health? a pilot study. Adm Policy Ment Health 2010; 37: 388-98. 
16 Salyers MP, Tsai J, Stultz TA. Measuring recovery orientation in a hospital setting. Psychiatr Rehabil J 2007; 31: 131-7.

17 Kraus SW, Stein $\mathrm{CH}$. Recovery-oriented services for individuals with mental illness and case managers' experience of professional burnout. Community Ment Health J 2013; 49: 7-13.

18 Hungerford $C$, Kench $P$. The perceptions of health professionals of the implementation of recovery-oriented health services: a case study analysis. J Ment Health Train Educ Pract 2013; 8: 208-18.

19 Salas E, Burke CS, Cannon-Bowers JA. Teamwork: emerging principles. Int J Manage Rev 2000; 2: 339-56.

20 Pascaris A, Shields LR, Wolf J. The work and recovery project: changing organizational culture and practice in New York City outpatient services. Psychiatr Rehabil J 2008; 32: 47-54.

$21 \mathrm{McHugh} P$, Byrne $M$. The teamworking challenges of care planning. Ir J Psychol Med 2012; 29: 185-9.

22 Leamy M, Clarke E, Le Boutillier C, Bird V, Choudhury R, MacPherson R, et al. Recovery practice in community mental health teams: national survey. $\mathrm{Br} \mathrm{J}$ Psychiatry 2016; 209: 340-6.

23 Lemieux-Charles L, McGuire WL. What do we know about health care team effectiveness? A review of the literature. Med Care Res Rev 2006; 63: 263300

24 Maynard MT. The Impact of Experience and Familiarity: An Examination of Project Teams. Doctoral Dissertation, Business Administration, Management, University of Connecticut, 2007.

25 Mathieu J, Maynard TM, Rapp T, Gilson L. Team effectiveness 1997-2007: a review of recent advancements and a glimpse into the future. $J$ Manage 2008; 34: 410-76.

26 Ilgen DR, Hollenbeck JR, Johnson M, Jundt D. Teams in organizations: from input-process-output models to IMOI models. Annu Rev Psychol 2005; 56: 517-43.

27 Scammon DL, Tabler J, Brunisholz K, Gren LH, Kim J, Tomoaia-Cotisel A, et al. Organizational culture associated with provider satisfaction. J Am Board Fam Med 2014; 27: 219-28

28 O'Connell M, Tondora J, Croog G, Evans A, Davidson L. From rhetoric to routine: assessing perceptions of recovery-oriented practices in a state mental health and addiction system. Psychiatr Rehabil J 2005; 28: 378-86.

29 Rosenberg D, Svedberg P, Schon UK. Establishing a recovery orientation in mental health services: evaluating the Recovery Self-Assessment (RSA) in a Swedish context. Psychiatr Rehabil J 2015; 38: 328-35.

30 Williams J, Leamy M, Bird V, Harding C, Larsen J, Le Boutillier C, et al. Measures of the recovery orientation of mental health services: systematic review. Soc Psychiatry Psychiatr Epidemiol 2012; 47: 1827-35.

31 Rossi ME. The development and validation of the comprehensive team interdependence scale. MA Thesis, Department of Psychology, University of South Florida, 2008.

32 Spreitzer GM. Social structural characteristics of psychological empowerment Acad Manage J 1996; 39: 483-504.

33 Campion MA, Papper EM, Medsker GJ. Relations between work team characteristics and effectiveness: a replication and extension. Pers Psychol 1996; 49: 429-52.

34 De Dreu CKW. Cooperative outcome interdependence, task reflexivity, and team effectiveness: a motivated information processing perspective. J Appl Psychol 2007; 92: 628-38.

35 Bock GW, Zmud RW, Kim YG, Lee JN. Behavioral intention formation in knowledge sharing: examining the roles of extrinsic motivators, social-psychological forces, and organizational climate. MIS Q 2005; 29: 87-111.
36 Chiocchio F, Lebel P, Dube JN. Informational role self-efficacy: a validation in interprofessional collaboration contexts involving healthcare service and project teams. BMC Health Serv Res 2016; 16: 1-7.

37 Jehn KA, Mannix EA. The dynamic nature of conflict: a longitudinal study of intragroup conflict and group performance. Acad Manage J 2001; 44: 23851.

38 Song $M$, Xie J. Does innovativeness moderate the relationship between cross-functional integration and product performance? Int Mark Rev 2000; 8: $61-89$

39 Chiocchio F, Grenier S, O'Neill A, Savaria K, Willms JD. The effects of collaboration on performance: a multilevel validation in project teams. Int J Proj Organisat Manag 2012; 4: 1-37.

40 Sicotte C, D'Amour D, Moreault MP. Interdisciplinary collaboration within Quebec community health care centres. Soc Sci Med 2002; 55: 991-1003.

41 Spector PE. Measurement of human service staff satisfaction: development of the Job Satisfaction Survey. Am J Community Psychol 1985; 13: 693-713.

42 Meyer JP, Allen NJ, Topolnytsky L. Commitment in a changing world of work. Can Psychol 1998; 39: 83-93.

43 Simons TL, Peterson RS. Task conflict and relationship conflict in top management teams: the pivotal role of intragroup trust. Am J Community Psychol 2000; 85: 102-11.

44 Anderson NR, West MA. Measuring climate for work group innovation: development and validation of the team climate inventory. J Organ Behav 1998; 19: 235-58.

45 Chiocchio F. Indicateurs Pertinents à la Collaboration dans le Milieu de la Santé: Cadre Conceptuel et Inventaire de Mesures [Relevant Indicators for Collaboration in the Healthcare Milieu: Conceptual Framework and Inventory of Measures]. Université de Montréal, 2012.

46 Dale H, Lee A. Behavioural health consultants in integrated primary care teams: a model for future care. BMC Fam Pract 2016; 17: 1-9.

47 Kidd SA, McKenzie K, Collins A, Clark C, Costa L, Mihalakakos G, et al. Advancing the recovery orientation of hospital care through staff engagement with former clients of inpatient units. Psychiatr Serv 2014; 65: 221-5.

48 Webster L, Hackett RK. Burnout and leadership in community mental health systems. Admin Policy Ment Health 1999; 26: 387-99.

49 Markon MP, Chiocchio F, Fleury MJ. Modelling the effect of perceived interdependence among mental healthcare professionals on their work role performance. J Interprof Care 2017; 31: 520-8.

50 Körner M, Lippenberger C, Becker S, Reichler L, Müller C, Zimmerman L, et al. Knowledge integration, teamwork and performance in health care. $J$ Health Organ Manage 2016; 30: 227-43.

51 Hülsheger UR, Anderson N, Salgado JF. Team-level predictors of innovation at work: a comprehensive meta-analysis spanning three decades of research. $J$ Appl Psychol 2009; 94: 1128-45.

52 Thiebaut GC, Farand L, Fleury MJ. Policies and mental health action plans in OECD: lessons for Quebec? Sante Ment Que 2014; 39: 65-84.

53 Fleury MJ, Grenier G, Robitaille D. Implementation of the consultation-liaison model in Quebec and its impact on primary care providers. Ment Health Fam Med 2016; 12: 228-40.

54 Foster T. Suicide prevention as a prerequisite for recovery from severe mental illness. Int J Psychiatry Med 2013; 46: 15-25.

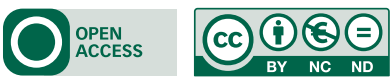

